\title{
Effet de la diversité générationnelle sur le besoin en espaces verts. L'indice de Shannon outil de lecture, de mesure et d'évaluation
}

Efeito da diversidade geracional na necessidade de espaços verdes. Ferramenta de leitura, medição e avaliação do índice de Shannon

Effect of generational diversity on the need for green spaces. Shannon's index reading, measurement and evaluation tool

Toufik Djedi[a] (1)

[a] Université Constantine 3 - Salah Boubnider, Faculté d'architecture et d'urbanisme, Département d'architecture, Constantine, Algérie

Comment citer: Djedi, T. (2021). Effet de la diversité générationnelle sur le besoin en espaces verts. L'indice de Shannon outil de lecture, de mesure et d'évaluation. urbe. Revista Brasileira de Gestão Urbana, 13, e20200037. https://doi.org/10.1590/2175-3369.012.e20200037

\section{Resume}

Le présent article vise à éclaircir l'effet de la diversité générationnelle sur la formulation du besoin d'espaces verts. L'outil de lecture adopté est le l'indice de Shannon. Son utilisation est accompagnée par l'indice de richesse (nombre de tranches d'âges) et l'indice d'équitabilité. Il s'avère que, plus la composition de la population est variée et équilibrée, plus le besoin en espaces verts par habitant occupe une position médiane en termes de surface d'espaces verts par habitant. Une « bonne diversité » au sens de richesse et équitabilité se révèle ainsi parmi les ferments pour une présence soutenue et préservation de l'élément végétal dans les projets d'aménagement.

Mots clés: Diversité générationnelle. Espaces verts. Indice de Shannon. Indice d'équitabilité. Projets d'aménagement.

\section{Resumo}

Este artigo tem como objetivo esclarecer o efeito da diversidade geracional na formulação da necessidade de espaços verdes. A ferramenta de leitura adotada é o índice de Shannon. Seu uso é acompanhado pelo índice de riqueza (número de faixas etárias) e pelo índice de justiça. No entanto, quanto mais variada e equilibrada a composição da população, a necessidade de espaço verde por habitante ocupa uma posição mediana em termos da necessidade de área verde por habitante. Uma "boa diversidade" no sentido de riqueza e justiça é revelada entre as enzimas para uma presença e uma preservação sustentada do elemento vegetal nos projetos de desenvolvimento.

Palavras-chave: Diversidade geracional. Espaços verdes. Índice de Shannon. Índice de justiça. Projetos de desenvolvimento.

\begin{abstract}
This article aims to clarify the effect of generational diversity on the formulation of the need for green space. The reading tool adopted is the Shannon index. Its use was accompanied by the Richness Index
\end{abstract}

TD est architecte et urbaniste, e-mail: dtk2073@yahoo.fr 
(number of age groups) and the Equitability Index. However, the more varied and balanced composition of the population, the need for green space per inhabitant occupies a median position in terms of the need for green space per inhabitant. A "good diversity" in the sense of richness and equity is revealed among the ferments for a sustained presence and preservation of the plant element in development projects.

Keywords: Generational diversity. Green spaces. Shannon index. Equitability index. Planning projects.

\section{Introduction}

Les espaces verts urbains jouent un rôle important dans l'amélioration de la qualité de vie dans les villes (Kaplan, 2001; Kaplan \& Austin, 2004; Wells, 2000; Korpela \& Hartig, 1996; Chiesura, 2004; Tyrvainen et al., 2005, 2007). L'aménagement, la conception, la gestion et la protection des espaces verts urbains figurent en tête des priorités de la «durabilité» des établissements humains pour améliorer la qualité de leurs environnements locaux aujourd'hui (Baycan-Levent et al., 2002).

La littérature académique souligne de nombreux avantages procurés par les espaces verts et ouverts qui couvrent les dimensions économiques, sociales et environnementales. Ces espaces peuvent réduire de nombreux coûts liés à la maintenance des infrastructures urbaines en réduisant les inondations, la poussière, en refroidissant les zones chaudes et en réduisant la vitesse du vent et les dégâts causés par les tempêtes. Développer les espaces verts dans les zones urbaines renforce également les habitats urbains, augmente la biodiversité et renforce la connectivité écologique (Byrne \& Sipe, 2010).

Considérant le fait qu'être en contact avec la nature est réputé être bénéfique pour un individu à bien des égards, y compris pour les jeunes, les modèles de planification des espaces ouverts urbains contemporains, révèlent que les paramètres quantitatifs (taille de la population, localisation spatiale et distance) sont les mesures les plus couramment utilisées pour déterminer les espaces ouverts. Les avantages de ces derniers sont principalement déterminés par la quantité et la qualité de ces zones, ainsi que par leur accessibilité (Tyrvainen et al., 2005).

L'approche par la demande ou par «standards», qui se traduit de différentes manières: ratio d'espace ouvert par tranche de population (généralement pour 1000 habitants), pourcentage de sol à réserver pour les espaces ouverts, rayon d"influence ou de service.et normes propre à une zone mais aussi les normes de dimensions (Veal, 2013), a connue une expansion dans le monde entier depuis son introduction au 19ème siècle grâce à cette mise en œuvre simple . A l'inverse de l'approche par l'offre (déterminisme écologique, réserves de biosphère, paysages protégés, modèles paysagers) dont le processus et conduites sont complexes et appellent à une haute connaissance de l'écologie, contrairement aussi au modèle opportuniste qui n'est guère considéré comme un outil de planification systématique en raison de l'absence de principes de planification, et que les opportunités étant largement dues au hasard. L'approche par la demande se révèle très appropriée à l'échelle locale (Maruani \& Amit-Cohen, 2007).

Bien que ces normes aient été inscrites dans les meilleures pratiques et même dans certaines législations, elles ne répondent pas nécessairement aux besoins des résidents. L'indifférence de l'approche à l'égard des systèmes sociaux et environnementaux, où un ensemble de règles est appliqué uniformément à toutes les situations fait d'elle l'objet de critiques (Byrne \& Sipe, 2010; Harnik,2009; Harnik \& Simms, 2004; Taylor, 1999; Wilkinson,1985).

De nos jours, le passage d'un urbanisme moderne à un urbanisme participatif, à fait émerger de nouvelles approches et méthodes d'évaluation plus soucieuses de la qualité: «Approche personnalisée », évaluation "fondée sur les besoins », reconnaissant les différences et que différentes personnes ont des «besoins» très différents d'accès aux espaces verts urbains et que des solutions novatrices peuvent satisfaire à ces exigences (Byrne \& Sipe, 2010). À l'exemple des groupes de discussions, méthode de sociotope...etc. Des approches qui considèrent non seulement le nombre absolu de personnes dans une zone géographique donnée, mais aussi et surtout leur composition sociodémographique, leurs préférences en matière de loisirs passives et actives et celles de divers sous-groupes au sein de cette 
population (Buttram, 1990; Anderson \& Heyne, 2000; Thompson, 2002; Smoyer-Tomic et al., 2004; Ståhle, 2009; Byrne \& Sipe, 2010).

\section{Contexte général du questionnement}

La ville du vingt-et-unième siècle, face aux besoins de répondre à la variété, évolution et changements rapides des besoins et modes d'habiter, est encline à assumer la diversité qui commence à en façonner le devenir. Cette dernière devient une réalité incontournable pour l'aménagement urbain aujourd'hui, en réponse aux rythmes accélérés des changements d'une société complexe, plus rationnelle, plus individualiste et plus différenciée, donnant naissance à des « profils » de vie et de consommation de plus en plus différenciés, rendant de moins en moins perceptible l'appartenance éventuelle à des groupes sociaux. Cette diversification sociale transforme également les structures familiales et leur fonctionnement (Ascher, 2013).

Des changements qui constituent un défi aux élus et agences d'urbanismes qui souhaitent satisfaire les besoins et préférences des habitants en termes d'approche par besoins pour ce qui est de la demande d'espaces verts. malgré le fait que le comportement de loisirs est façonné par la race et l'âge (Crespo et al., 2000; Riddick \& Stewart, 1994). Peu d'études ont intégré le rôle de l'âge dans la formation des préférences et des besoins en matière de loisirs au sein de populations diverses (Payne et al., 2002). Les quelques études qui existent ont documenté les différences de préférences en matière de paysagères entre adolescents et adultes (Kaplan \& Talbot, 1988), les différences dans la participation à des loisirs en plein air (Dwyer, 1994; Dwyer \& Marsinko, 1997), et des changements dans la nature générale du comportement de loisirs à un age plus avancé (Smale \& Dupuis, 1993; Freysinger \& Stanley, 1995; Robinson \& Godbey, 1997; Payne et al., 2002).

L'étude des différences entre les groupes d'âge peut donc éclairer la manière dont l'âge, par rapport à d'autres données sociodémographiques, contribue aux variations dans les attitudes et les comportements en matière de loisirs. L'âge peu ainsi être l'un des meilleurs prédicateurs du besoin perçu de terrains supplémentaires et de la fréquence des visites (Payne et al., 2002). Notre contribution essaye de déceler les impacts d'une " bonne » diversité générationnelle, dans le sens de richesse et équitabilité de distribution des groupes d'âges sur le besoin en surfaces pour des espaces verts urbains. Le questionnement est alors comme suit. Quel est l'effet de la diversité générationnelle sur le besoin en surface pour des espaces verts?, Cependant, les différences culturelles, visions de la ville et des approches d'études des espaces verts entre approche par «standards »et « approche adaptée » nous conduit à préciser le choix du support à retenir pour l'étude de l'effet de la diversité générationnelle pour le besoin en surfaces d'espaces verts.

L'étude retient la surface de $10 \mathrm{M}^{2}$ par personne, un minimum recommandé par l'Organisation Mondiale de la Santé (OMS). un support largement partagé par l'ensemble de la communauté internationale, qui apparait dans deux approches vraiment opposées dans l'étude du rapport au «vert » en ville, l'approche par «standards » ou par besoins et l'approche de Sociotope (Ståhle, 2009). Une ville où la nature en est l'antithèse et un modèle considérant la nature comme une composante fondamentale de la ville à l'instar des capitales des pays nordiques tels que Stockholm. Cette recommandation n'est retenue dans un premier temps de l'étude que comme support de lecture de l'effet de la diversité générationnelle sur les besoins en surface.

Ce choix nous place d'emblée dans une position et culture de laboratoire, en dehors de toutes autres influences (situation habitantes, revenu, patrimoine, classes sociales, pratiques habitantes, ...etc.) et enjeux suivant le contexte de chaque projet, qui peuvent s'imposer comme variables intermédiaires d'explication du rapport.Ce qui permet de tester dans un premier temps l'effet de la composition des groupes d'âges sur la possibilité de présence du végétal. Un deuxième scénario nous permet de voir l'effet du changement des préférences traduites sous formes de ratios sur le besoin en espaces verts. Un 
troisième scénario quant à lui va s'atteler à explorer l'effet de l'augmentation des tranches d'âges par redistribution de la tranche d'âge des adultes sur le besoin en espaces vert.

\section{Méthodologie}

L'âge étant associé à des attitudes et des préférences, notre étude adopte une perspective double pour comprendre (l'effet des chaque variable isolement) les effets des deux variables: âges et préférences exprimées en ratio par âge sur l'évaluation des besoins de surfaces en espaces verts. Dans cette approche, l'étude de la variation renseigne sur le pouvoir prédictif de chaque variable (l'âge et préférences) pour expliquer la variation de la variable dépendante, le besoin en surface d'espaces verts.

La première partie de la contribution est une lecture où il s'agira d'éclaircir l'effet de la diversité de la distribution des groupes d'âges sur le besoin (la demande) en espaces verts. Tenant compte des préférences et demandes en surfaces par tranches d'âges comme variable intermédiaire. Supposant des ratios d'usages constants et une structure changeante de population. L'étude retient la décomposition du ratio de $10 \mathrm{M}^{2}$ par tranches d'âges proposée dans le contexte français comme support, vue la rareté des études et documents qui proposent d'autres interprétations de la décomposition de ce ratio par tranche d'age (Tableau 1). Un ratio repris par le schéma directeur de la région Île-de-France 2013 est loin d'être couvert. À Paris, $90 \%$ des arrondissements sont en dessous des $10 \mathrm{~m}^{2}$, dont $70 \%$ en dessous des $5 \mathrm{~m}^{2}$ (L'institut Paris Region, 2015).

Tableau 1. Décomposition du ratio de $10 \mathrm{M}^{2} / \mathrm{Hab}$. suivant les normes françaises

\begin{tabular}{ccccc}
\hline $\begin{array}{c}\text { Tranche } \\
\text { d'âge }\end{array}$ & $\begin{array}{c}\text { \% de la } \\
\text { population }\end{array}$ & $\begin{array}{c}\text { Surface/ } \\
\text { usager }\end{array}$ & Ratio & Descriptions \\
\hline 0-4 ans & $0.110 \%$ & $02.0 \mathrm{M}^{2}$ & $0.2 \mathrm{M}^{2}$ & Jardins d'enfants \\
5-9 ans & $0.110 \%$ & $08.0 \mathrm{M}^{2}$ & $0.8 \mathrm{M}^{2}$ & Jardins d'enfants \\
10-14 ans & $0.110 \%$ & $20.0 \mathrm{M}^{2}$ & $2.0 \mathrm{M}^{2}$ & Plaines de jeux \\
$15-19$ ans & $0.110 \%$ & $20.0 \mathrm{M}^{2}$ & $2.0 \mathrm{M}^{2}$ & Plaines de jeux \\
Adultes & $0.660 \%$ & $8.33 \mathrm{M}^{2}$ & $5.0 \mathrm{M}^{2}$ & $\begin{array}{c}\text { Promenades des adultes, promenades et aires de } \\
\text { repos familiales, aires sablées pour jeux libres }\end{array}$ \\
Total & $1.0100 \%$ & & $10 \mathrm{M}^{2}$ & \\
\hline
\end{tabular}

Source: Auteurs d'après circulaire du 8 février 1973 relative à la politique d'espaces verts (France, 1973, p. 15-16). Ministère de l'équipement (France, 1992), L'aménagement des espaces verts, le Moniteur, Paris (France, 1973, 19-20).

À partir d'un programme sur le logiciel Matlab version R2013, les scénarios sont établis sur la base de combinaisons des tranches d'âges avec un taux de variation par pan de $5 \%$ à chaque fois. Un premier scénario étudie l'effet de la variation des taux des quatre tranches d'âges dans les compositions démographiques. Le deuxième scénario considère l'effet de la variation des compositions démographiques sur le besoin en espaces verts suivant un changement du ratio d'espaces verts par tranche d'âge. Supposant en premierune augmentation des ratios par tranches d'âges. Une lecture de l'effet de la variation des compositions démographiques avec ces nouveaux ratios sur le besoin en espaces verts s'ensuit.

Ce deuxième scénario est motivé par les résultats des recherches consacrées aux différences des préférences/comportements des activités en matière de loisirs en fonction de la race, âges, localisation, ... etc. tels que celles de Payne et al. (2002) et de Dwyer (1994) ainsi que celle de Crespo et al. (2000). Et les dernières tendances en matière de planification des parcs proposant d'avoir davantage d'espaces verts non conventionnels et d'utilisations alternatives des espaces verts existants. Où polyvalence et souplesse des espaces verts est de rigueur afin de maintenir les tendances actuelles, mais aussi les activités futures, qui pourraient ne pas permettre de prévoir avec exactitude (Grose, 2009; Lavery,1975; Maruani \& Amit-Cohen,2007).

Par ailleurs, l'hypothèse testée de Floyd \& Shinew (1999) sur l'effet du contact interracial sur la modération, nivellement et aplatissement des différences en matière de loisir, et les constats de Scott et 
Willits (1998) sur la stabilité du répertoire et engagement des loisirs au cours des différents stades de la vie, nous oriente à favoriser un scénario, où «prévoir l'imprévisible est de voir «grand» (Durand, 2017) on ce sens on prévoit par un doublement des ratios (Tableau 2). Par ailleurs, pour les projets d'urbanisme actuels, dont les réponses sont désormais bien différentes et ne sauraient se suffire du chiffrable (Durand, 2017). l'étude en explorant l'effet de la variété envisage et favorise du « mou», absent à ce chiffrable.

Tableau 2. Ratios par usager pour le scénario du doublement des besoins en surfaces d'espaces verts

\begin{tabular}{cccc}
\hline Tranche d'âge & \% de la population & Surface/ usager & Ratio \\
\hline 0-4 ans & $0.110 \%$ & $04.00 \mathrm{M}^{2}$ & $0.4 \mathrm{M}^{2}$ \\
$5-9$ ans & $0.110 \%$ & $16.00 \mathrm{M}^{2}$ & $1.6 \mathrm{M}^{2}$ \\
10-14 ans & $0.110 \%$ & $40.00 \mathrm{M}^{2}$ & $4.0 \mathrm{M}^{2}$ \\
$15-19$ ans & $0.110 \%$ & $40.00 \mathrm{M}^{2}$ & $4.0 \mathrm{M}^{2}$ \\
Adultes & $0.660 \%$ & $16.66 \mathrm{M}^{2}$ & $10 . \mathrm{M}^{2}$ \\
Total & $1.0100 \%$ & & $\mathbf{2 0 \mathbf { M } ^ { 2 }}$ \\
\hline
\end{tabular}

Source: Auteurs suivant scénario du doublement du ratio de $10 \mathrm{M}^{2}$ par habitant d'après la circulaire du 8 février 1973 relative à la politique d'espaces verts (France, 1973, p. 15-16). Ministère de l'équipement, 1992 (France, 1992), L'aménagement des espaces verts, le Moniteur, Paris (France, 1973, 19-20).

Pour le troisième scénario, une redistribution de la tranche d'âge des adultes suivant trois cohortes est opérée afin d'explorer l'effet du nombre de tranches d'âges sur la formulation du ratio par habitant.

L'outil de lecture adopté pour évaluer l'effet de la diversité sur le besoin d'espaces verts est le coefficient de Shannon: $H^{\prime}=-\sum_{I=1}^{S} P i \log 2 P i$ (ROSELT/OSS, 2004).

Un indice dérivé de la théorie de l'information. Avec H': indice de Shannon, $\mathrm{S}$ nombre des tranches d'âges (richesse). $\mathrm{Pi}=\mathrm{nj} / \mathrm{N}$ fréquence relative des tranches d'âges (proportion d'espèce i). Avec nj fréquence relative de la tranche d'âge $\mathrm{j}$ dans l'unité d'échantillonnage et $\mathrm{N}$ somme des fréquences relatives spécifiques. Sensible aux espèces (tranche d'âge) relativement rares. Son utilisation et interprétation est complétée par l'indice de richesse: nombre de tranches d'âges $S$ et l'indice d'équitabilité E (équirépartition) correspondant à l'indice de Shannon avec $E=H^{\prime} / \log _{2} S$. (ROSELT/OSS, 2004).

Ces indices nous permettrons à travers les scénarios de modification des taux de tranches d'âges des compositions démographique et ratios de se profiler une idée sur les changements des besoins en espaces verts dans une partie de ville ou de quartier, voir un projet de quartier.

\section{Résultats}

Les principaux résultats obtenus de la mise en rapport du besoin d'espaces vert avec les indices d'équitabilité et de Shannon. Obtenus à partir d'un programme sur le logiciel Matlab version R2013,des scénarios retenus établis sur la base de combinaison des tranches d'ages avec un taux de variation par pan de 5\% à chaque fois. Sont représentés par les graphes des nuages de points sur les Figures 1, 2, 3, 4, 5, 6 .

\section{Scénario 1: effet des variations des taux des groupes d'âges}

Pour le rapport entre l'indice d'équitabilité et besoin d'espaces verts Figure1, le nuage de points prend une forme concave, qui devient plus soutenue lorsque l'indice dépasse la valeur 0.8. L'augmentation de la valeur de l'indice se traduit par une concentration de points et leur convergence vers le ratio de $10 \mathrm{M}^{2}$ par habitant qui est la médiatrice. On en déduit donc qu'avec une structure de population de plus en plus équilibrée, c'est-à-dire une répartition régulière des tranches d'ages, le besoin en espaces verts diminue. Par ailleurs, pour une même valeur de l'indice le ratio d'espaces verts par habitant n'est pas une valeur mais un intervalle dont la plage diminue avec l'augmentation de l'indice d'équitabilité. 


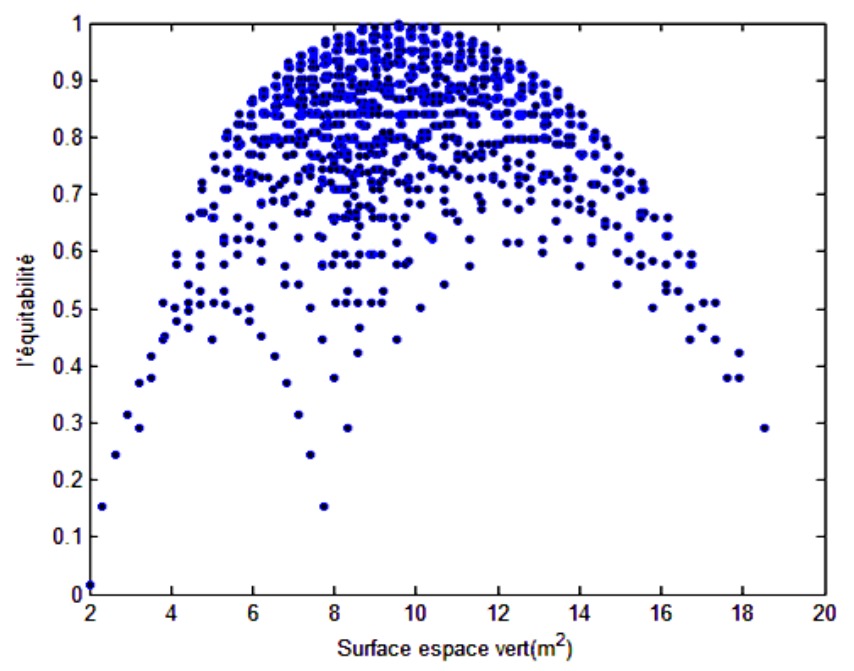

Figure 1 - Rapport entre Besoins d'espaces verts en $M^{2}$ et l'indice d'équitabilité. Source: Auteur.

Pour ce qui est du rapport de ratio d'espace vert avec l'indice de Shannon Figure 2,rappelons en premier que, la valeur maximale de l'indice de Shannon varie en fonction du nombre de tranche d'age et qu'elle ne sera que purement théorique du fait que les tranches d'âges ne sont pas présentes dans les mêmes proportions. Pour notre étude, l'augmentation de la valeur de l'indice de Shannon se traduit par une réduction du besoin d'espaces verts, plus particulièrement lorsque l'indice dépasse 1.7 le ratio est alors inclue dans un intervalle de $6.3 \mathrm{M}^{2}$ à $14.5 \mathrm{M}^{2}$. Obtenir le ratio de $10 \mathrm{M}^{2}$ est plus soutenue avec l'augmentation de la valeur de l'indice. Par ailleurs, une forte proportion des deux premières tranches d'âges fait diminuer le besoin de surface, à l'inverse, un fort pourcentage des tranches d'âges d'adolescents et adultes font augmenter les besoins en surfaces vertes. Ainsi, la zone médiane des valeurs de ratio d'espace vert se distingue par un équilibre de répartition des tranches d'âges.

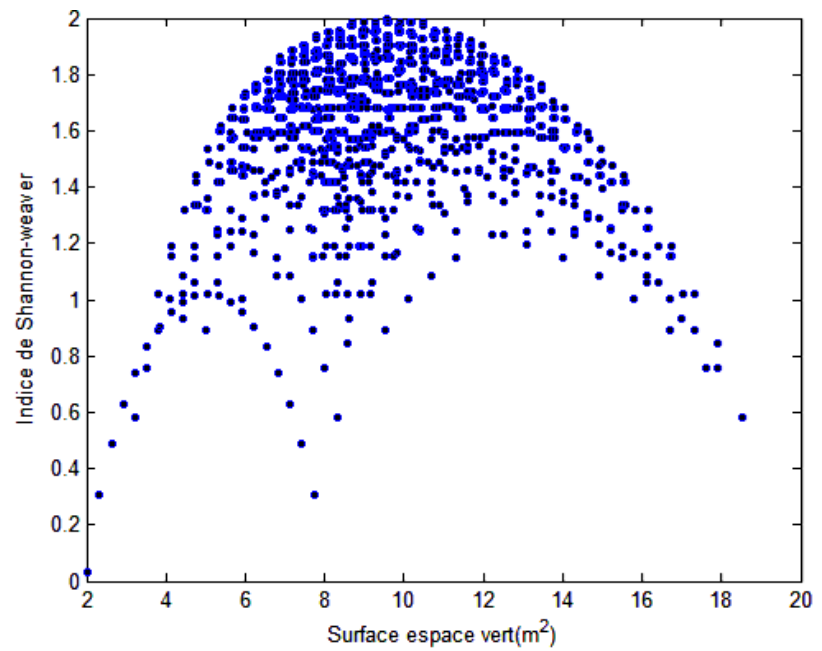

Figure 2 - rapport entre Besoins d'espaces verts en $M^{2}$ et l'indice de Shannon. Source: Auteur.

\section{Scénario 2: effet de variation des taux de groupes d'âges et augmentation des ratios par tranches d'âges}

Pour ce deuxième scénario du doublement du ratio d'espaces verts par tranche d'âge, le schéma du premier scénario du rapport entre indice d'équitabilité et besoins d'espaces verts est reproduit. Le nuage de points prend une forme concave, de l'augmentation de la valeur de l'indice résulte une réduction du 
besoin en surface d'espace vert. Toutefois, les intervalles d'espaces verts pour chaque valeur de l'indice d'équitabilité prennent une valeur double, par exemple pour la valeur 0.8 de l'indice le besoin en espace vert s'inscrit dans l'intervalle de $12.36 \mathrm{M}^{2}$ à $33.27 \mathrm{M}^{2}$ Figure 3. Quant à la valeur médiane elle est de $20 \mathrm{M}^{2}$.

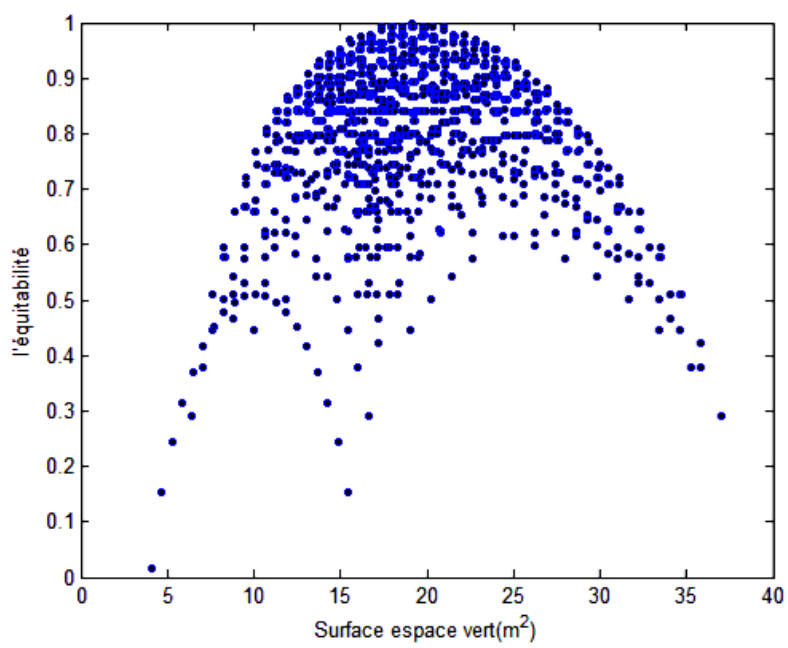

Figure 3 - Rapport entre Besoins d'espaces verts en $M^{2}$ et l'indice d'équitabilité. Source: auteur.

Quant à l'indice de Shannon Figure 4, On observe un profil du nuage de points sur le graphe semblant au premier scénario: réduction du besoin d'espace vert dûà l'augmentation de l'indice. L'obtention du ratio de $20 \mathrm{M}^{2}$ (médiatrice) est conditionnée par une augmentation de la valeur de l'indice. Une dominance des deux premières tranches d'âges (enfants de moins de dix ans) fait diminuer le besoin de surface, à l'inverse, un fort pourcentage des tranches d'âges d'adolescents et adultes font augmenter les besoins en surfaces vertes Figure 4 .

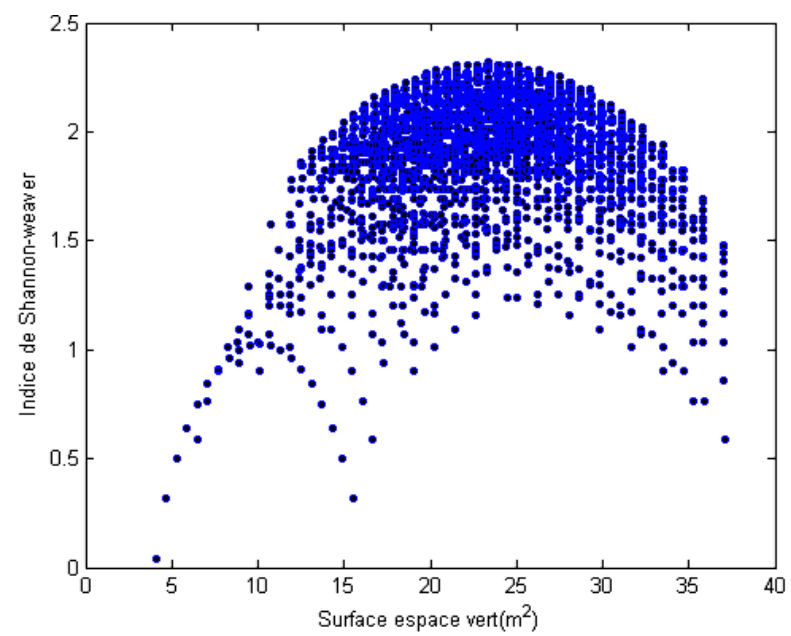

Figure 4 - Rapport entre Besoins d'espaces verts en $M^{2}$ et l'indice de Shannon. Source: Auteur.

\section{Scénario 3: Augmentation du nombre de tranches d'âges par redistribution de la tranche d'age des adultes}

Pour ce qui est du troisième scénario, augmentation du nombre de tranches d'âges par la proposition d'une redistribution de la tranche d'âge des adultes suivant 3 cohortes, où l'on retient un scénario de l'augmentation des besoins chez les adolescents de 15 à 19 ans et un maintient de la demande pour la récréation chez la première tranche des adultes de 20 à 39 ans avec une baisse progressive pour les tranches d'âges de 40 à 59 ans et plus de 60 ans (Tableau 3). 
Tableau 3. décomposition du ratio d'espaces verts suivant une redistribution de la tranche d'âge des adultes

\begin{tabular}{cccc}
\hline Tranche d'âge & \% de la population & Surface/ usager & Ratio \\
\hline 0-4 ans & $0.110 \%$ & $02.00 \mathrm{M}^{2}$ & $0.2 \mathrm{M}^{2}$ \\
$5-9$ ans & $0.110 \%$ & $08.00 \mathrm{M}^{2}$ & $0.8 \mathrm{M}^{2}$ \\
$10-14$ ans & $0.110 \%$ & $20.00 \mathrm{M}^{2}$ & $2.0 \mathrm{M}^{2}$ \\
$15-19$ ans & $0.110 \%$ & $30.00 \mathrm{M}^{2}$ & $3.0 \mathrm{M}^{2}$ \\
$20-39$ ans & $0.330 \%$ & $15.00 \mathrm{M}^{2}$ & $4.5 \mathrm{M}^{2}$ \\
$40-59$ ans & $0.220 \%$ & $10.00 \mathrm{M}^{2}$ & $2.0 \mathrm{M}^{2}$ \\
+60 ans & $0.110 \%$ & $04.00 \mathrm{M}^{2}$ & $0.4 \mathrm{M}^{2}$ \\
Total & $1.0100 \%$ & & $\mathbf{1 2 . 9 \mathbf { M } ^ { 2 }}$ \\
\hline
\end{tabular}

Source: Auteur suivant Tableau 1 et scénario auteurs pour les 3 dernières tranches d'âges.

Le rapport à l'indice d'équitabilité: Le nuage des points commencent à se resserré depuis la valeur 0.6 de l'indice, la forme parabolique quand à elle commence à se consolidé depuis la valeur 0.8 de l'indice, avec un déplacement de la médiane du ratio d'espaces verts vers une valeur qui se rapproche de $12.5 \mathrm{M}^{2}$ lorsque la valeur de l'indice dépasse 0.7 les mêmes observations que les deux premiers scénarios sont à retenir, le ratio n'est pas une valeur mais un intervalle, l'équirépartition des tranches d'âges tend à réduire le besoin en espaces verts. Toutefois, on enregistre une augmentation et extension des valeurs des intervalles des surfaces d'espaces verts. Figure 5.

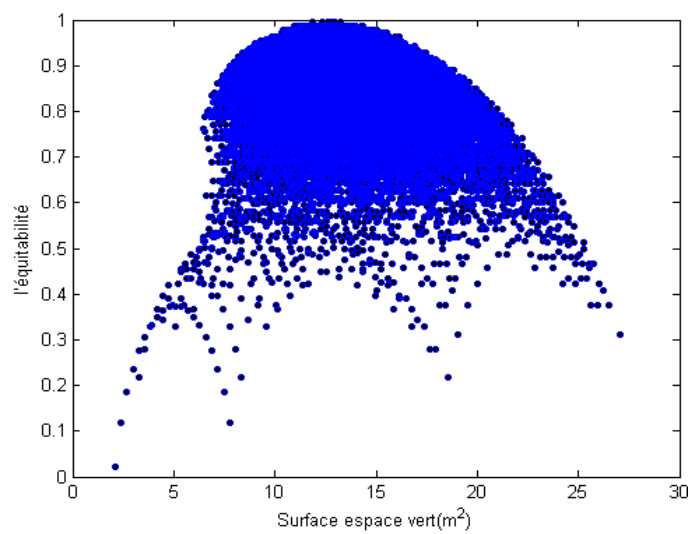

Figure 5 - Rapport entre besoins d'espaces verts et l'indice d'équitabilité. Source: Auteur.

Rapport à l'indice de Shannon Figure 6: on observe que la valeur de l'indice passe à plus de 2.75 suite à l'augmentation du nombre de groupes d'âges. Les mêmes constats que les deux scénarios précédents sont à retenir. A savoir diminution du besoin avec l'augmentation des valeurs. Le changement du nombre de cohortes dans la distribution consolide le nuage de points à partir de la valeur 1.5 de l'indice.

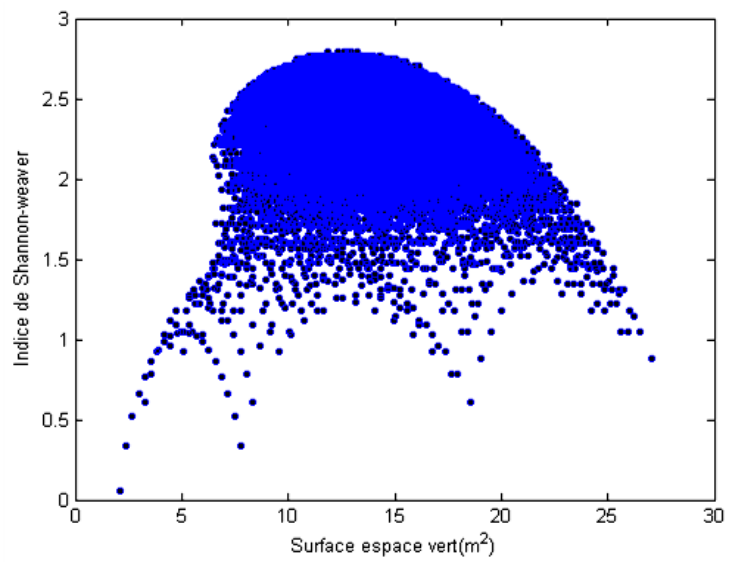

Figure 6 - Rapport entre besoins d'espaces verts et indice de Shannon. Source: Auteur. 


\section{Discussion}

L'étude de l'apport de la diversité pour une présence du végétal, suivant les deux composantes, richesse et équitabilité .faisant usage de l'indice d'équitabilité et l'indice de Shannon, affirme qu'une " meilleure » diversité générationnelle est garante d'une présence soutenue et préservation de l'élément végétal dans l'aménagement urbain. En effet l'équitabilité de distribution des tranches réduit le besoin en espace vert dont la plage des valeurs tend vers une valeur médiane avec l'augmentation de la valeur de E. Il s'agit donc d'approcher une plage de distribution avoisinant une meilleure équitabilité. Une intention faisable et abordable, au vue des changements des régimes de vie, de différenciation et individualisation qui caractérisent la société à l'aube du troisième millénaire (Ascher, 2013). Signalons que l'effet positif d'une «Bonne diversité» est dû à l'effet des ratios par classe d'âge différents permettant l'optimisation de la présence des espaces verts. La position de la valeur médiatrice du ratio est une résultante du nombre de cohorte et ratio par tranche d'âge.

Les résultats obtenus nous permettent d'être à l'abri de «l'hypothèse de compensation», qui veut que les résidents compenseront le mauvais accès aux espaces verts privés en utilisant des espaces verts publics dans les milieux denses. Mais loin aussi de l'un des paradoxes de consolidation urbaine ou densification, qui est de stimuler les déplacements basés sur les loisirs, car les citadins cherchent à s'évader à la campagne ou vers d'autres lieux de loisirs et d'expériences récréatives.

En ce sens, les résultats, viennent contrecarrer la notion simpliste, selon laquelle plus de parcs et donc de surfaces sont nécessaires lorsque la densité de population est accrue. Notion omettant de tenir compte des caractéristiques et besoins différents en matière d'espaces verts des résidents de quartiers à «forte densité», car il n'existe pas de " résident de densité supérieure» typique. Par ailleurs, les résultats converges avec les conclusions des travaux de Nathalie Cadiou et Vincent Fouchier sur la présence végétale dans la ville nouvelle d'EVRY, qui devant la complexité des perceptions et des usages des espaces de nature, où les pratiques concrètes paraissent déterminantes, pose la question de l'intérêt du critère quantitatif qui reste si souvent employé fixant un seuil à atteindre n'ayant qu'une valeur plus hygiénique que sensible (Cadiou \& Fouchier,1997). L'étude concorde aussi avec les recommandations de Jason Byrne et Neil Sipe dans leur revue de la littérature de la planification des espaces verts et ouverts pour la consolidation urbaine (2010), pour ce qui est de l'importance à accorder au critère qualitatif plus que quantitatif.

\section{Conclusion}

La présente contribution vise à éclaircir l'effet de la diversité générationnelle sur la formulation du besoin d'espaces verts. L'outil de lecture adopté est le coefficient de Shannon. Son utilisation est appuyée par l'indice de richesse (nombre tranches d'âges) et l'indice d'équitabilité. Il s'avère que, plus la composition de la population est variée et équilibrée, plus le besoin en espaces verts par habitant occupe une position médiane en termes de besoin de surface d'espaces verts par habitant.

Une « bonne diversité » tenant compte d'une « forte » valeur de la richesse et de la valeur de l'indice d'équitabilité de la composition démographique, retenant un plus grand nombre de tranches d'âges pour la structure démographique et une équirépartition des individus des différentes tranches d'âges, se révèle salvatrice pour une présence soutenue de l'élément végétal dans les projets d'aménagement de quartiers résidentiels. Par ailleurs, la contribution révèle qu'il y lieu d'aborder la demande d'espace vert par intervalle et plage de valeurs et non par valeur fixe, ce qui permet et annonce un rapprochement entre approche quantitatives (par standard) et approches qualitatives à l'instar des approches personnalisées tels que les groupe de discussion et méthode de sociotope....etc. Reconnaissant les différences et que différentes personnes ont des «besoins» très différents d'accès aux espaces verts urbains. 
Cependant, les résultats sont relatifs et limités ayant un caractère spéculatif. Bien que différents scénarios d'usages des espaces verts par les différentes cohortes d'utilisateurs aient été établis, par les ratios choisis par tranche d'âge. Ces derniers sont le plus souvent tributaires de plusieurs paramètre d'études et d'enquêtes, variant d'un contextes à un autre: taux de participation par activité et groupe d'âge, groupe racial et ethnique, le sexe, différence culturelles, évolution des préférences sur les activités en plein air, régimes de loisir existant et émergents, mode de vie, composition du ménage, situation familiale de la personne, éducation, niveau de revenus, handicap, origine rurale ou urbaine de la personne...etc.

En effet, «la demande de nature», présente un caractère polysémique. Chaque urbain à sa propre définition de la nature. Si les enquêtes récentes ont confirmé l'importance accordée aux espaces verts urbains (Boutefeu, 2009; Bourdeau-Lepage et al., 2012), elles ne permettent guère de vérifier que ce désir d'espaces verts correspond systématiquement à un désir de nature ni, lorsque c'est le cas, de savoir de quelle nature il s'agit. La satisfaction du désir de nature en ville apparait aujourd'hui encore plus qu'hier comme une composante clef du bien-être de l'Homme (Bourdeau-Lepage \& Vidal, 2014). Devenu un homo urbanus. Il cherche alors «[...] disposer des agréments de la ville tout en satisfaisant son désir de nature et de calme [...]» (Bourdeau-Lepage, 2012, p. 2) et devient un homo qualitus du moins dans les sociétés occidentales (Bourdeau-Lepage \& Vidal, 2014).

Bien que, l'équirépartition des classes d'âges de la composition démographique de la population dans un quartier ou secteur reste difficile à atteindre. L'approche d'un état plus ou moins équilibré est possible, au vue d'une société hypertexte, de plus en plus rationnelle et plus différenciée. Et une différenciation sociale de plus en plus complexe. Menant à une diversification sociale qui transforme également les structures familiales et leur fonctionnement (Ascher, 2013).

L'enjeu est de taille pour un développement urbain durable conciliant demande de nature et urbanisation durable. Si l'approche par tranches d'âges permet d'être au plus près et à l'écoute de la demande et moins éloignée de la réalité. Une «bonne» diversité générationnelle amoindri les plages de valeur du besoin permettant ainsi une cohabitation entre nature, jardinage et espace public sans risque de s'exclure l'un l'autre. La mise en concurrence entre espaces verts et réserves de nature « sauvage » en ville dont la fermeture au public pourrait donc avoir pour conséquence de contribuer à l'étalement urbain, ne devient plus une fatalité et se voie alléger par cette réduction d'une offre qui vient en réponse au contexte.

Une «bonne» diversité générationnelle nous met aussi à l'abri de la mobilité de compensation que l'on trouve dans les grandes métropoles et qui remet en question les qualités en matière de durabilité de ces villes. Et à l'abri de l'un des paradoxes de consolidation urbaine, stimulant les déplacements basés sur les loisirs, car les citadins cherchent à s'évader temporairement à la campagne ou vers d'autres lieux de loisirs et d'expériences récréatives. Le rapport simpliste selon lequel forte densité de population équivaux à plus de surface de parcs se voie contrecarrer, car ne tenant pas en compte des besoins et demandes différentes en matière d'espaces verts des citadins.

Notre contribution vise aussi à sensibiliser l'opinion des différents acteurs impliqués dans le processus du projet d'habitat, pouvoir public, aménageurs, promoteurs, designers, associations et habitants, sur le potentiel de la diversité générationnelle comme gage pour une amélioration de la qualité des quartiers résidentiels en termes de préservation et présence végétale. Les indices de Shannon, de richesse et d'équitabilité comme moyen de lecture et de mesure nous à permis un tant soit peu de faire le lien entre pensées écologiques et pratiques urbanistiques.

Une éventuelle intégration de ces indices dans la boite à outils des urbanistes et aménageurs à des fins d'aide à la décision n'est pas à négliger, pour améliorer l'équité environnementale, redresser les inégalités et éviter l'aggravation de la privation relative par une plus grande réflexivité. D'autre part, l'adaptation des actions à entreprendre aux évolutions temporelles pour le maintient de taux de couverture végétale satisfaisant, évaluation des besoins dans les nouveaux projets de quartiers résidentiels suivant leurs compositions démographiques et l'orientation des arbitrages sur la 
morphologie à retenir, figurent parmi les éventuelles possibilités d'usage de l'indice de Shannon $H^{\prime}$ et l'indice d'équitabilité E correspondant à l'indice de Shannon.

\section{Références}

Anderson, L., \& Heyne, L. (2000). A statewide needs assessment using focus groups: perceived challenges and goals in providing inclusive recreation services in rural communities. Journal of Park and Recreation Administration, 18(4), 17-37.

Ascher, F. (2013). Les nouveaux principes de l'urbanisme suivi de Lexique de la ville plurielle (pp. 27-94). Avignon: Édition de l'aube.

Baycan-Levent, T., Van Leeuwen, E., Rodenburg, C., \& Nijkamp, P. (2002, 21-26 de september). Development and management of green spaces in European cities: a comparative analysis. In Proceedings of the 38th International Planning Congress on "The Pulsar Effect" Planning with Peaks (pp. 237-247). Amsterdam: vrije Universiteite.

Bourdeau-Lepage L., Langlois, W., \& Sablé, T. (2012). La nature en ville. Espaces verts et bien-être, Enquête menée auprès de 150 personnes à Lyon au printemps. Université lyon3.

Bourdeau-Lepage, L. (2012). La ville miroir de nos société. In L. Bourdeau-Lepage (Ed.), Regards sur la ville (GÉOGRAPHIE) (pp. 1-6). Paris: Economica Anthropos.

Bourdeau-Lepage, L., \& Vidal, R. (2014). Comprendre la demande sociale de nature en ville. In: C. Chomarat-Ruiz (Ed), Nature urbaine en projets (pp. 37-52.). Archibooks.

Boutefeu, E. (2009). La demande sociale de nature en ville. Enquête auprès des habitants de l'agglomération lyonnaise. Revue Urbia, no 8, 21-38.

Buttram, J. L. (1990). Focus groups: a starting point for needs assessment. The American Journal of Evaluation, 11(3), 207-212. http://dx.doi.org/10.1177/109821409001100305.

Byrne, J., \& Sipe, N. (2010). Green and open space planning for urban consolidation: a review of the literature and best practice (pp. 1-59). Brisbane: Griffith University Urban Research Program.

Cadiou, N., \& Fouchier, V. (1997). La présence végétale dans la ville nouvelle d'Évry. Des usages et appropriations diversifiés. Annales de la Recherche Urbaine, 74, 95-102.

Crespo, C. J., Smit, E., Andersen, R. E., Carter-Pokras, O., \& Ainsworth, B. E.(2000). Race/ethnicity, social class and their relation to physical inactivity during leisure time: Results from the Third National Health and Nutrition Examination Survey, 1988-1994. American Journal of Preventive Medicine, 18(1), 46-53.

http://dx.doi.org/10.1016/S0749-3797(99)00105-1. PMid:10808982.

Chiesura, A. (2004). The role of urban parks for the sustainable city. Landscape and Urban Planning, 68(1), 129138. http://dx.doi.org/10.1016/j.landurbplan.2003.08.003.

Durand, A. (2017). Mutabilité urbaine la nouvelle fabrique des villes.Français: Éditions Infolio.

Dwyer, J. F. (1994). Customer diversity and the future demand for outdoor recreation (General Technical Report RM252, 58 p.). Fort Collins, CO: Roccky Mountain Forest and Range Experiment Station, U.S. Department of Agriculture, Forest Service.

Dwyer, J. F., \& Marsinko, A. (1997). Trends in participation rates for wildlife-associated outdoor recreation activities by age and race/ethnicity: Implications for cohort-component projection models. In H. Vogelsong (Ed.), Proceedings of the 1997 Northeastern Recreation Research Symposium (pp. 252-256). Radnor: U.S. Department of Agriculture, Forest Service, Northeastern Forest Experiment Station.

Floyd, M. F., \& Shinew, K. (1999). Convergence and divergence in leisure style among Whites and African Americans: toward an interracial contact hypothesis. Journal of Leisure Research, 31(4), 359-384.

http://dx.doi.org/10.1080/00222216.1999.11949872. 
France. Comité Interministériel d'action Pour la nature et l'environnement. (1973). Circulaire du 8 février 1973 relative à la politique d'espaces verts. Paris: Journal officiel de la République Française, p. 15-16.

France. Ministère de l'équipement, du Logement, des Transports et du Tourisme. (1992). L'amenagement des espaces verts: conception technique et réalisation Dossiers d'études et de travaux Modalités administratives. (pp. 1920). Paris: Ministère de l'équipement, du Logement, des Transports et du Tourisme.

Freysinger, V. J., \& Stanley, D. (1995). The impact of age, health, and sex on the frequency of older adults' leisure activity participation: a longitudinal study. Activities, Adaptation and Aging, 19(3), 31-42.

http://dx.doi.org/10.1300/J016v19n03_03.

Grose, M. (2009). Changing relationships in public open space and private open space in suburbs in south-western Australia. Landscape and Urban Planning, 92(1), 53-63. http://dx.doi.org/10.1016/j.landurbplan.2009.02.006.

Harnik, P. (2009). Shoehorn parks. Landscape Architecture Magazine, May, 42.

Harnik, P., \& Simms, J. (2004). Parks: how far is too far? Planning Magazine, 70(11), 8-11.

Kaplan, R. (2001). The nature of the view from home: psychological benefits. Environment and Behavior, 33(4), 507542. http://dx.doi.org/10.1177/00139160121973115.

Kaplan, R., \& Austin, M. E. (2004). Out in the country: sprawl and the quest for nature nearby. Landscape and Urban Planning, 69(2-3), 235-243. http://dx.doi.org/10.1016/j.landurbplan.2003.09.006.

Kaplan, R., \& Talbot, J. (1988). Ethnicity and preference for natural settings: a review and recent findings. Landscape and Urban Planning, 15(1-2), 107-117. http://dx.doi.org/10.1016/0169-2046(88)90019-9.

Korpela, K., \& Hartig, T. (1996). Restorative qualities of favourite places. Journal of Environmental Psychology, 16(3), 221-233. http://dx.doi.org/10.1006/jevp.1996.0018.

Lavery, P. (1975). The demand for recreation: a review of studies. The Town Planning Review, 46(2), 185-200. http://dx.doi.org/10.3828/tpr.46.2.6827351272m636j6.

L'institut Paris Region. (2015). Ile-de-France 2030: la region se transforme rdecouvrir la nature en Ville (Les carnets pratiques du SDRIF, No. 6). Paris: L'institut Paris Region.

Maruani, T., \& Amit-Cohen, I. (2007). Open space planning models: a review of approaches and methods. Landscape Urban Plann, 81(1), 1-13. http://dx.doi.org/10.1016/j.landurbplan.2007.01.003.

Payne, L. L., Mowen, A. J., \& Orsega-Smith, E. (2002). An examination of park preferences and behaviors among urban residents: the role of residential location, race and age. Leisure Sciences, 24(2), 181-198. http://dx.doi.org/10.1080/01490400252900149.

Réseau d'Observatoires de Surveillance Ecologique à Long Terme - ROSELT/OSS. l'Observatoire du Sahara et du Sahel - OSS. (2004). Contributions Techniques CT No. 14. Documentd'appui No. 2. Fiche technique No. 8. Tunisia: ROSELT.

Riddick, C., \& Stewart, D. G. (1994). An examination of life satisfaction and importance of leisure in the lives of older females: a comparison of Blacks to Whites. Journal of Leisure Research, 26(1), 75-87.

http://dx.doi.org/10.1080/00222216.1994.11969945.

Robinson, J. P., \& Godbey, G. (1997). Time for life: the surprising ways Americans use their time. University Park, PA: The Pennsylvania State University Press.

Scott, D., \& Willits, F. (1998). Adolescent and adult leisure patterns: A reassessment. Journal of Leisure Research, 30(3), 319-330.

Smale, B. J. A., \& Dupuis, S. L. (1993). The relationship between leisure activity participation and psychological well-being across the lifespan. Journal of Applied Recreation Research, 18(4), 281-300.

Smoyer-Tomic, K. E., Hewko, J. N., \& Hodgson, M. J. (2004). Spatial accessibility and equity of playgrounds in Edmonton, Canada. The Canadian Geographer. Geographe Canadien, 48(3), 287-302.

http://dx.doi.org/10.1111/j.0008-3658.2004.00061.x. 
Ståhle, A. (2009). Dense + Verte = Ville durable., Ploemeur (pp. 1-91). Ploemeur: Au délor. Récupéré le 8 septembre 2009, de https://fr.slideshare.net/audelor/actess-sociotopes-web3

Taylor, D. E. (1999). Central Park as a model for social control: urban parks, social class and leisure behavior in Nineteenth-Century America. Journal of Leisure Research, 31(4), 420-477.

http://dx.doi.org/10.1080/00222216.1999.11949875.

Thompson, C. (2002). Urban open space in the 21st century. Landscape and Urban Planning, 60(2), 59-72. https://doi.org/10.1016/S0169-2046(02)00059-2.

Tyrvainen, L., Makinen, K., \& Schipperijn, J. (2007). Tools for mapping social values of urban woodlands and other green spaces. Landscape and Urban Planning, 79(1), 5-19. http://dx.doi.org/10.1016/j.landurbplan.2006.03.003.

Tyrvainen, L., Pauleit, S., Seeland, K., \& de Vries, S. (2005). Benefits and uses of urban forests and trees. In C. Konijnendijk, K. Nilsson, T. B. Randrup, \& J. Schipperijn (Eds.), Urban forests and trees (pp. 81-114). Berlin, Heidelberg: Springer. http://dx.doi.org/10.1007/3-540-27684-X_5.

Veal, A. J. (2013). Open space planning standards in Australia: in search of origins. Australian Plants, 50(3), 224232. http://dx.doi.org/10.1080/07293682.2012.739567.

Wells, N. M. (2000). At home with nature. Effects of 'greenness' on children's cognitive functioning. Environment and Behavior, 32(6), 775-795. http://dx.doi.org/10.1177/00139160021972793.

Wilkinson, P. (1985). The golden fleece: the search for standards. Leisure Studies, 4(2), 189-203. http://dx.doi.org/10.1080/02614368500390141.

Éditeur: Rodrigo Firmino

Reçu: Févr. 09, 2020

Approuvé: Aoû† 06, 2020 